\title{
The Royal College of Pathologists of Australasia (RCPA) position statement on COVID-19
}

Ivan Bastian, Lynette Waring

The Royal College of Pathologists of Australasia (RCPA) this week released a position statement on COVID-19 immunoglobulin (Ig) G/IgM rapid point-of-care tests. ${ }^{1}$ The statement states:

- Molecular testing on a single throat with deep nasal swab is the current test of choice for the diagnosis of acute COVID-19 infection;

- COVID-19 IgG/IgM rapid tests have no role to play in the acute diagnosis of COVID-19 virus infection, and most importantly;

- COVID-19 IgG/IgM rapid tests will miss patients in the early stages of disease when they are infectious to other people.

One COVID-19 IgG/IgM rapid test received extensive media publicity throughout Australia and New Zealand recently. However, these IgG/IgM tests have a fundamental limitation. They rely on the detection of antibodies made by the patient in response to SARS-CoV-2, the virus that causes COVID-19. They do not detect the virus and thus must not be used to screen for early infection.

Patients may only make antibodies to COVID-19 infection a week to 12 days after they first become sick (Figure 1). If doctors rely on COVID-19 IgG/IgM/ IgA rapid tests early in the disease, their diagnosis will be wrong. Furthermore, elderly or immunocompromised patients may never (or only much later) develop anti-SARS-CoV-2 antibodies.

Reliable detection of anti-IgM antibodies early in infection is also problematic because of cross-reactions resulting in false-positive results. Most importantly from a public health perspective, COVID-19-positive patients are infectious to other people soon after infection when the COVID-19 IgG/ IgM tests give false-negative results. Australia's public health response will be compromised by the use of these tests in the early stages of COVID-19 disease.

In sharp contrast, the basic strength of molecular tests is that they directly detect gene sequences of the virus in the early stages of infection when the patient is infectious. More than 250,000 SARS-CoV-2 tests have been undertaken in Australia so far. In fact, Australia is among the world leaders for the number of SARS-CoV-2 molecular tests performed per million population. New Zealand pathology laboratories are also performing large numbers of tests, with more than 23,000 tests performed to date. No laboratory test is perfect. Molecular tests can miss COVID-19 infections if a poor sample is collected or if the patient carries a low level of virus. A well-collected single throat with deep nasal swab is the optimal sample for polymerase chain reaction (PCR). Limited data are available on the presence/duration of viraemia (virus in the blood) and therefore on the performance of PCRs (molecular tests) on blood samples.

The RCPA Quality Assurance Programs (RCPAQAP) have just run one of the first quality assurance programs for SARS-CoV-2 in the world. This program will help laboratories optimise their molecular tests, which have had to be developed rapidly in response to the COVID-19 pandemic.

\section{Disease and reaction time}

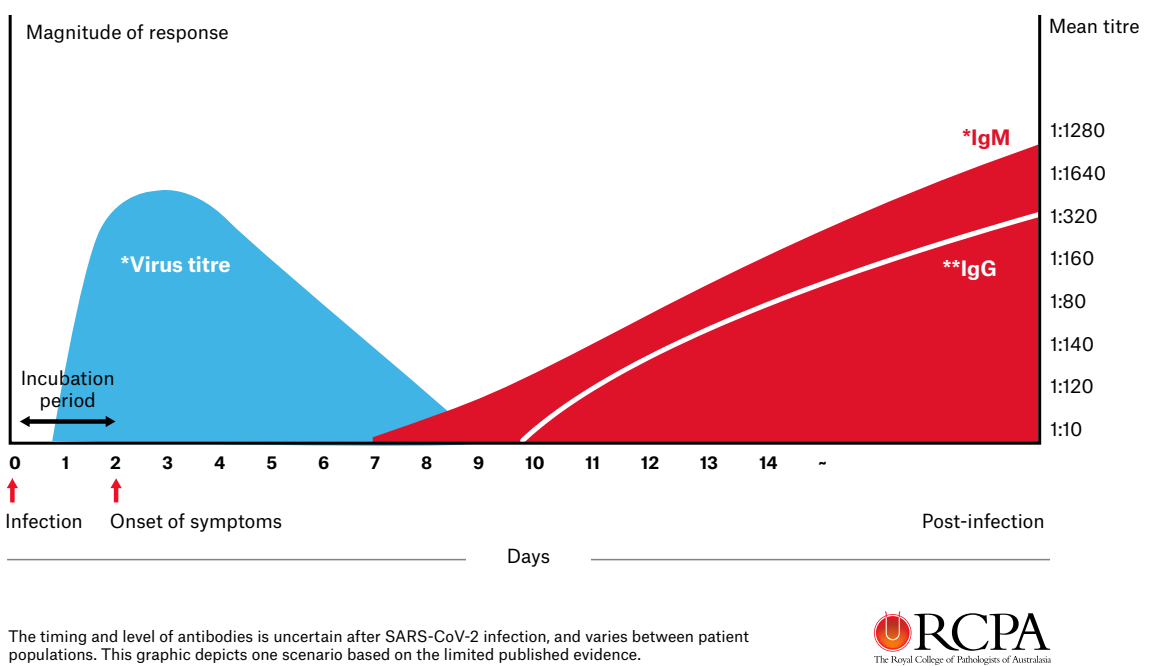

Figure 1. A scenario based on limited published evidence of the timing and level of antigen and antibody response for the SARS-CoV-2 infection 
For the detection of early COVID-19 disease, RCPA supports the use of molecular tests for SARS-CoV-2 and strongly opposes the introduction of COVID-19 IgG/IgM rapid tests for this purpose. The excellent laboratory response to the COVID-19 pandemic in Australia would be jeopardised by inappropriate widespread use of COVID-19 IgG/IgM rapid tests.

These antibody tests may have a place in detecting unrecognised past infection and immunity; however, that role needs to be rigorously evaluated. The RCPA is aware that further review and assessment of these tests has been commissioned by the Therapeutic Goods Administration (TGA), and the RCPA and its Fellows look forward to working with the TGA and other stakeholders to determine the best use of these COVID-19 IgG/IgM rapid tests in the Australian setting.

Finally, the TGA requires that all SARS-CoV-2 tests kits, both molecular and antibody based, are subject to an effective evaluation process in Australia. This is especially important during the COVID-19 emergency, when the risk to our community of a false-negative test is very high.

The position statement can be found at the RCPA website (www.rcpa.edu. au/Library/COVID-19-Updates/

Media-Releases).

First published online 15 April 2020.

\section{Authors}

Ivan Bastian MBBS, PhD, FRCPA, Clinical Director Microbiology Infectious Diseases Directorate, SA

Pathology, SA; Councillor, RCPA South Australia, SA

Lynette Waring MBBS, FRCPA, Director of

Microbiology, Melbourne Pathology, Vic; Chair,

RCPA Microbiology Advisory Committee, NSW

Competing interests: None.

Provenance and peer review: Commissioned, peer reviewed.

Citation: Bastian I, Waring L. The Royal College of Pathologists of Australasia (RCPA) position statement on COVID-19. Aust J Gen Pract 2020;49 Suppl 9. doi: 10.31128/AJGP-COVID-09.

Contact: ceo@rcpa.edu.au

\section{Reference}

1. The Royal College of Pathologists of Australasia. Position statement: COVID19 IgG/lgM rapid POCT tests. Surry Hills, NSW: RCPA, 2020. 